\title{
Speeding up coral reef conservation with Al-aided automated image analysis
}

To the Editor - Artificial intelligence through machine learning is advancing the power of collecting and processing data for nature conservation ${ }^{1}$. Machine learning algorithms are applied to identify species ${ }^{2}$, predict extinction probabilities ${ }^{3}$ and assess fisheries global data ${ }^{4}$, and this technology could be employed in coral reef ecology studies to understand large-scale impacts, such as those caused by climate change.

Global mass coral-bleaching events have been continuous and severe in the past decade ${ }^{5}$. Some regions are currently experiencing the third mass bleaching in five years, an average interval that is less than half what it was few decades ago, preventing the full recovery of coral reefs ${ }^{5}$. Large-scale monitoring of coral reefs, as is done for the Great Barrier Reef ${ }^{6}$, requires significant investment in collecting and processing data. Ecological studies of coral reefs are traditionally performed through photo-quadrats (where photographs of a standardized area are analysed) and underwater visual censuses (UVC) (where divers count fish in a standardized area $)^{7}$. Photo-quadrats analyses are extremely time-consuming, and UVCs involve significant sampler bias ${ }^{8}$. Alternatively, recent studies based on deep learning convolutional neural networks showed advantages of automated image analysis for fish and coral reefs studies ${ }^{2,9}$, revealing that data processing and reporting can be accelerated 200-fold and cost a fraction of the previous methods ${ }^{9}$. These recent experiments showed accuracies between $94 \%$ and $97 \%$ when compared to expert results.

A large-scale programme based on automated image analysis could speed up and standardize information, which could enable continuous tracking of impacts. This is necessary because of the current short time intervals between mass bleaching events and the fast pace of change in the ecological community structure in coral reefs worldwide ${ }^{5,10}$. This programme could allow the uploading of pictures, videos and environmental information by citizens and scientists through a user-friendly website and mobile application. Two valuable types of data that could be initially targeted are: (1) species-driven images, focusing on one or more species; and (2) community structure images, quantifying the local diversity.

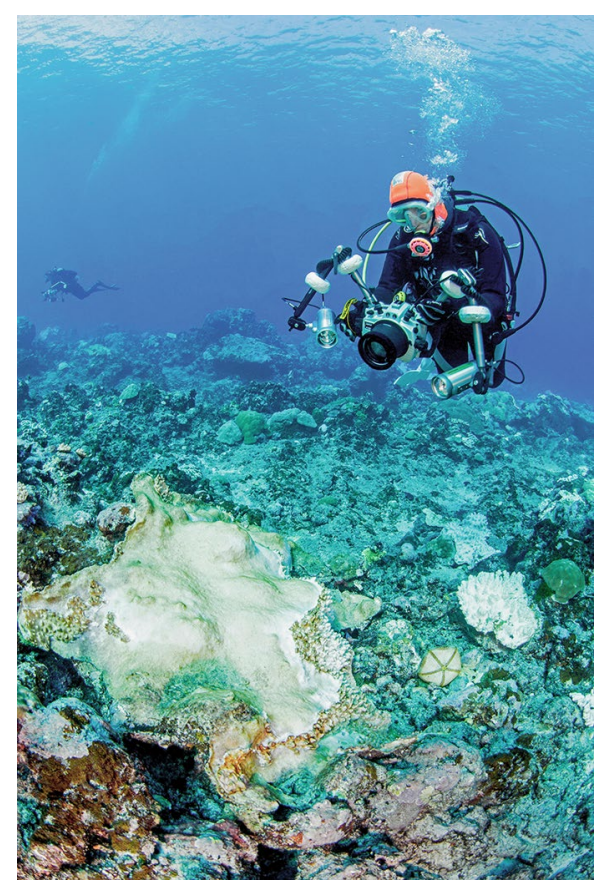

Credit: Herbert Frei/mauritius images $\mathrm{GmbH} /$ Alamy Stock Photo

Automated analysis of uploaded images and information could result in distributional maps of species, groups of species or richness levels, as well as biodiversity checklists per location, which is similar to what is offered by the iNaturalist platform. Coral reef communities could be assessed through general photographs of the seascape, or by using techniques such as stationary images (cameras on tripod-like structures), baited remote underwater videos (where bait is used to attract large fish to remote cameras) or video transects (where divers record communities along an established distance) focusing on mobile (pelagic and demersal fish) or benthic organisms (for example, corals, sponges and algae). Information about the community structure (for example, proportion of species, ecological groups and endangered species) and health status (for example, intensity of bleaching, diseases and sedimentation) could be visualized in maps and in time series, with the possibility of sorting by location or components of the coral reef community.

This large-scale programme based on automated image analysis could be implemented following successful initiatives that have mobilized thousands of volunteers and experts to monitor coral reefs around the world, such as the Reef Check and Reef Life Survey projects. However, the advantages of storing and processing data via cloud computing ${ }^{11}$ are numerous, especially regarding quicker data upload, visualization and sharing, which could be boosted by social media. The partnership between institutions and between specialists in artificial intelligence and those in coral reefs biodiversity is essential for the creation of such programme. To get involved, artificial intelligence researchers could contribute to ongoing ideas and programmes such as AI4Corals, Corail, CoralNet and iNaturalist, among others, helping to create user-friendly systems that could allow integration among projects, and easy sharing and interpretation of data.

A modern programme based on artificial intelligence could quickly quantify lethal and sub-lethal coral bleaching, coral reef recovery, phase shifts events, and effects of marine protected areas and restoration projects. The fast release of results is an important outcome for policymakers, who need trustworthy data to orient their decisions, and for scientists, who need data from a widespread area to better understand how climate change is affecting the ecology of coral reefs.

\section{José Anchieta C. C. Nunes (D) gor C. S. Cruz (D)2 , André Nunes ${ }^{3}$ and Hudson T. Pinheiro (D) 4 M \\ ${ }^{1}$ Reef Ecology Group, Salvador, Brazil. ${ }^{2}$ Federal University of Bahia, Salvador, Brazil. ${ }^{3}$ Zagow Technology Research, Salvador, Brazil. ${ }^{4}$ California Academy of Sciences, San Francisco, CA, USA. $\bigotimes_{e-m a i l: h t p i n h e i r o @ g m a i l . c o m}$}

Published online: 8 June 2020 https://doi.org/10.1038/s42256-020-0192-3

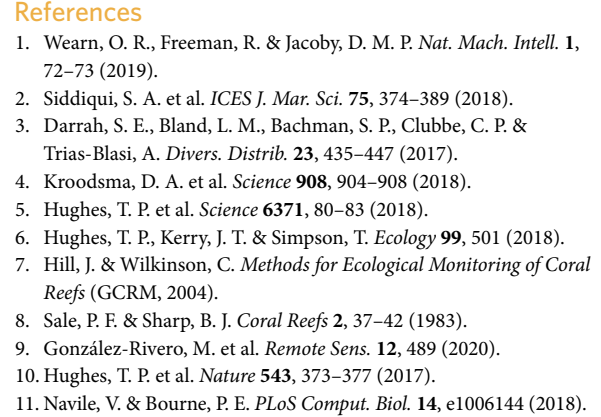

The authors declare no competing interests. 